\title{
Helicobacter pylori in both the sinuses and the stomach*
}

\author{
Paulo Borges Dinis', Teresa Matos ${ }^{1}$, Miguel Sardinha², Pedro Luís Alves², \\ Joana Vital ${ }^{3}$, Ana Maria Carvalho ${ }^{4}$, Jorge Vítor ${ }^{5}$ \\ 'Otolaryngology Department, CHLN, Lisbon, Portugal \\ 2 Faculty of Sciences, Lisbon University, Lisbon, Portugal \\ ${ }^{3}$ CQBFarma, Lisbon, Portugal \\ ${ }^{4}$ Pathology Department, CHLC-Curry Cabral Hospital, Lisbon, Portugal \\ ${ }^{5}$ Faculty of Pharmacy, Biochemistry and Human Biology Department, Lisbon University, Lisbon, Portugal
}

Rhinology Online, Vol 1: 194 - 200, 2018

http://doi.org/10.4193/RHINOL/18.035

*Received for publication:

July 24, 2018

Accepted: October 26, 2018

Published: November 22, 2018

\begin{abstract}
Background: The role played by Helicobacter pylori in the sinuses, and its association with the same organism's gastric infection, are still unclear.

Methods: In order to compare H.pylori colonization patterns in the nose and stomach we conducted a cohort analysis of 14 patients, eligible for sinus surgery due to chronic medically refractory rhinosinusitis, who were tested for simultaneous presence of $H$. pylori, by histology, culture and polymerase chain reaction, in pathologic sinus tissue collected during surgery and in gastric mucosa obtained through gastroduodenal endoscopy.
\end{abstract}

Results: H. pylori DNA was found in the sinus mucosa of $15.4 \%$ of patients with chronic rhinosinusitis, and all of them showed concurrent $H$. pylori stomach infection. Sinus colonization was not found without simultaneous gastric colonization, although most patients with gastric infection did not have the bacterial DNA in their sinuses. H. pylori's presence in the nose was not associated with local inflammatory status, and no cultures could be obtained from any of the sinus tissue samples, including those positive for H. pylori DNA.

Conclusions: Only H. pylori DNA, and not the culturable active form of the microorganism, could be found in the sinus mucosa of some patients with $H$. pylori gastric infection. We could not find evidence, however, that the bacterium's presence in the nose contributes to local mucosal inflammation.

Key Words: chronic rhinosinusitis, Helicobacter pylori, gastric infection

\section{Introduction}

Helicobacter pylori DNA has been detected in a number of extragastric locations, such as the oral cavity, tonsils and adenoids $s^{(1,2)}$, and even the middle ear and paranasal sinuses ${ }^{(2-15)}$. However, the significance of the microorganism's presence at these aero- digestive and respiratory sites is still unclear. It is believed that the oral cavity is an important reservoir for H. pylori, that contributes to the oral-oral route of transmission and acts as a source of stomach re-infection ${ }^{(1)}$. Others suggest that the bacterium may be capable of causing damage to the aero-digestive and respiratory mucosa in the same way it does to the gastric mu$\operatorname{cosa}^{(1)}$. However, this capacity for extra-gastric disease remains unproved, and some authors argue that it even seems unlikely.
In order to initiate tissue damage, not only does $H$. pylori require a set of events unique to the gastric milieu, but also culturable active forms of $H$. pylori have only been recovered outside the stomach in the aero-digestive tract in the vicinity of the upper esophagus (i.e. in tracheal secretions in intubated patients), and never as far away as the bronchi, lung, or the upper respiratory tract $^{(2)}$. That far from the stomach, only the microorganism's DNA suggests the presence of $H$. pylori at such locations.

We conducted the present investigation in order to see how $\mathrm{H}$. pylori colonization patterns in the nose and stomach are associated in chronic rhinosinusitis patients, as a way to also provide information on the eventual existence of an interaction between two seemingly unconnected pathologies, one in the digestive 
tract and the other in the upper respiratory tract. At the same time, we assessed if this known gastric pathogen has the capacity to also inflict direct injury to the sinus mucosa.

\section{Materials and Methods}

\section{Patients}

A total of 14 adult patients, 8 male and 6 female, aged 30 to 68 years (mean 48.1 years), requiring endoscopic sinus surgery due to medically recalcitrant chronic rhinosinusitis, with and without nasal polyposis, were enrolled in a consecutive, unselected manner. Informed consent was obtained from each patient, and the study protocol was approved by the institutional ethics committee. All patients had their biochemical and hematological profiles within the normal range, with the exception of mild-tomoderate eosinophilia in some atopic patients. Patients with cystic fibrosis, immotile cilia syndrome, and known immunodeficiencies were excluded. The inflammatory nature of the disease was subsequently confirmed by histopathologic examination in all patients enrolled.

Drug therapy for any underlying condition was allowed, including systemic steroids for asthma management. Antibiotic use in the 3 months prior to surgery was also noted. Moreover, patients were specifically asked about their history of prior H. pylori infection diagnosis and treatment, as well as current medication with antacids, histamine 2 -receptor antagonists, prokinetic agents, or proton-pump inhibitors (PPI's).

\section{Procedures}

In the immediate pre-operative period (from 1 to 10 days preop), all subjects underwent a 13C-labelled urea breath test. Surgery was performed under general anesthesia, with orotracheal intubation. Routine vasoconstriction of the nasal mucosa was carried out to minimize intra-operative bleeding, including infiltration, at critical sites, with 1\% xylocaine with 1:100,000 epinephrine.

The extent of the pathology on the computed tomography scans determined which sites were to be surgically approached. All patients required bilateral procedures due to roughly symmetrical extension of the pathology, according to the Lund-McKay classification ${ }^{(16)}$. During surgery, tissue samples of diseased sinonasal mucosa were collected at the anterior ethmoid (including the uncinate process, ethmoidal bulla, and meatal portion of the middle turbinate), and the posterior ethmoid sinus from one, randomly chosen, nasal cavity. The collected sinus specimens were subjected to: 1 ) histopathological examination, to assess the overall degree of local inflammation (graded as $0+$, no inflammation; $1+$, mild; $2+$, moderate; and $3+$, severe inflammation, depending on the inflammatory infiltrate cell density $\left.{ }^{(17)}\right)$; 2) microbiological culture, to isolate H. pylori; and 3) polymerase chain reaction (PCR) to amplify genomic H. pylori DNA. Enrolment required that the patients also agree to undergo a simultaneous upper gastrointestinal endoscopy, irrespectively of digestive symptoms. Biopsies were taken from the stomach mucosa lining, either from endoscopic-assessed suspected sites or iteratively, from the lesser or greater curvature of the antrum and the greater curvature of the corpus. Patients underwent gastroduodenal endoscopy either simultaneously with the sinonasal mucosa sampling (intra-operatively, under general anesthesia, while waiting for the vasoconstriction effect to occur in the nose) or up to two weeks post sinus surgery. The collected gastric biopsy specimens from the two sites, the antrum and the corpus, were analyzed by: 1) histopathology, to assess the overall degree of local inflammation (graded as $0+$, absent; $1+$, mild; $2+$, moderate; $3+$, severe inflammation, depending on the inflammatory infiltrate cell density); 2 ) histology to identify $H$. pylori in Warthin-Starry silver stained gastric mucosal biopsies, (also graded as $0+$, no bacteria found; $1+$, mild ; $2+$, moderate; $3+$, severe density colonization); and 3) microbiological culture of gastric biopsy specimens specific for $H$. pylori.

All sinus and stomach tissue samples were collected aseptically, transported to the laboratory at $4^{\circ} \mathrm{C}$ in Portagerm pylori (bioMérieux, France), and processed less than four hours after collection. For microbiological culture, a drop of tissue macerate was plated onto H. pylori-selective medium (Brucella supplemented with $10 \%$ horse blood and Brucella supplemented with $10 \%$ horse blood and Oxoid $H$. pylori-selective supplement (Dent) ) and incubated at $37^{\circ} \mathrm{C}$ in a microaerophilic atmosphere (Campygen, Oxoid) for up to 15 days. Colonies were then tested for urease, catalase and oxidase, and motility and were stained with Gram stain.

\section{Polymerase chain reaction}

For PCR testing, two sets of primers targeting the $16 \mathrm{~S}$ rRNA and $23 \mathrm{~S}$ rRNA genes of $H$. pylori were used, chosen to maximize detection as together they target sequences common to virtually all $H$. pylori strains ${ }^{(18,19)}$. The first primer pair, PCR-G, amplifies a 780bp fragment from the $16 \mathrm{~S}$ rRNA gene specific for the Helicobacter genus, Helico F - 5' CTATGACGGGTATCCGGC 3 ' and Helico R - 5' CTCACGACACGAGCTGAC $3^{\prime}{ }^{\prime(18)}$. The second primer pair, PCR-S, amplifies a $267 \mathrm{bp}$ fragment from the $23 \mathrm{~S}$ rRNA gene of the H. pylori, HPYS - 5' CGCATGATATTCCCATTAGCAGT $3^{\prime}$ and HPYA - $5^{\prime}$ AGGTTAAGAGGATGCGTCAGTC $3^{\prime \prime(19)}$. Ethidium bromide-stained agarose gel electrophoresis was employed for separating the PCR products according to their size. When interpreting the results, we considered a positive genus-specific PCR test a sign of presence of DNA for one of the various Helicobacter species, whilst the combination of a positive genus-specific with a positive species-specific PCR test was taken as a clear sign of presence of the actual $H$. pylori species. 
Table 1. Status of gastric and nasal inflammation and H. pylori infection for all patients.

\begin{tabular}{|c|c|c|c|c|c|c|c|c|c|c|}
\hline \multirow{3}{*}{$\begin{array}{c}\text { Patient } \\
1 \\
1\end{array}$} & \multirow{3}{*}{$\begin{array}{c}\text { Breath test } \\
\text { Pos }\end{array}$} & \multicolumn{4}{|c|}{ Stomach } & \multicolumn{5}{|c|}{ Sinus } \\
\hline & & \multirow{2}{*}{$\begin{array}{l}\text { Inflammation } \\
\text { Antrum: 2+ }\end{array}$} & \multirow{2}{*}{$\begin{array}{c}\begin{array}{c}\text { H. pylori } \\
\text { histology }\end{array} \\
\text { Antrum: 3+ }\end{array}$} & \multirow{2}{*}{$\begin{array}{c}\text { H. pylori } \\
\text { culture } \\
\text { Pos }\end{array}$} & \multirow{2}{*}{$\begin{array}{c}\text { H. pylori PCR } \\
\text { - G / -S } \\
\text { Pos / Pos }\end{array}$} & \multirow{2}{*}{$\begin{array}{c}\text { Inflammation } \\
2+\end{array}$} & \multirow{2}{*}{$\begin{array}{c}\text { H. pylori } \\
\text { culture } \\
\text { Neg }\end{array}$} & \multicolumn{3}{|c|}{$\begin{array}{l}\text { H. pylori PCR } \\
\text { - G / -S }\end{array}$} \\
\hline & & & & & & & & Pos & I & Pos \\
\hline & & Corpus: $2+$ & Corpus: $3+$ & Pos & Pos / Pos & & & & & \\
\hline \multirow[t]{2}{*}{2} & Neg & Antrum: NA & Antrum: NA & $\mathrm{Neg}$ & $\mathrm{Neg} / \mathrm{Neg}$ & $1+$ & $\mathrm{Neg}$ & Neg & / & $\mathrm{Neg}$ \\
\hline & & Corpus: NA & Corpus: NA & $\mathrm{Neg}$ & Neg / Pos & & & & & \\
\hline \multirow[t]{2}{*}{3} & Pos & Antrum: NA & Antrum: NA & NA & NA / NA & $1+$ & $\mathrm{Neg}$ & Pos & I & Neg \\
\hline & & Corpus: $1+$ & Corpus: $2+$ & Pos & Pos / Pos & & & & & \\
\hline \multirow[t]{2}{*}{4} & Pos & Antrum: $2+$ & Antrum: $0+$ & Pos & Pos / Pos & $1+$ & $\mathrm{Neg}$ & Pos & I & Neg \\
\hline & & Corpus: $1+$ & Corpus: $1+$ & Pos & Pos / Pos & & & & & \\
\hline \multirow[t]{2}{*}{5} & $\mathrm{Neg}$ & Antrum: $1+$ & Antrum: 0+ & $\mathrm{Neg}$ & Neg / Pos & $1+$ & $\mathrm{Neg}$ & Pos & I & $\mathrm{Neg}$ \\
\hline & & Corpus: $1+$ & Corpus: $0+$ & $\mathrm{Neg}$ & Neg / Pos & & & & & \\
\hline \multirow[t]{2}{*}{6} & Pos & Antrum: $2+$ & Antrum: $3+$ & Pos & Pos / Pos & $1+$ & $\mathrm{Neg}$ & Pos & I & $\mathrm{Neg}$ \\
\hline & & Corpus: $2+$ & Corpus: $2+$ & Pos & NA / Neg & & & & & \\
\hline \multirow[t]{2}{*}{7} & Pos & Antrum: NA & Antrum: NA & NA & NA/NA & $2+$ & $\mathrm{Neg}$ & $\mathrm{Neg}$ & / & Neg \\
\hline & & Corpus: NA & Corpus: NA & NA & NA/NA & & & & & \\
\hline \multirow[t]{2}{*}{8} & Pos & Antrum: $2+$ & Antrum: $3+$ & Pos & Pos / Pos & $2+$ & $\mathrm{Neg}$ & Pos & I & $\mathrm{Neg}$ \\
\hline & & Corpus: $1+$ & Corpus: $2+$ & Pos & Pos / Pos & & & & & \\
\hline \multirow[t]{2}{*}{9} & Pos & Antrum: 1+ & Antrum: 0+ & Neg & $\mathrm{Neg} / \mathrm{Neg}$ & $1+$ & $\mathrm{Neg}$ & Neg & I & Neg \\
\hline & & Corpus: $1+$ & Corpus: $0+$ & $\mathrm{Neg}$ & $\mathrm{Neg} / \mathrm{Neg}$ & & & & & \\
\hline \multirow[t]{2}{*}{10} & $\mathrm{Neg}$ & Antrum: $2+$ & Antrum: $1+$ & Pos & Pos / Neg & $2+$ & $\mathrm{Neg}$ & Pos & I & Neg \\
\hline & & Corpus: $2+$ & Corpus: $0+$ & Pos & NA / Pos & & & & & \\
\hline \multirow[t]{2}{*}{11} & Pos & Antrum: $2+$ & Antrum: $2+$ & Pos & Neg / Pos & $1+$ & Neg & $\mathrm{Neg}$ & I & $\mathrm{Neg}$ \\
\hline & & Corpus: $1+$ & Corpus: $0+$ & Pos & $\mathrm{Neg} / \mathrm{Neg}$ & & & & & \\
\hline \multirow[t]{2}{*}{12} & Neg & Antrum: 1+ & Antrum:0+ & $\mathrm{Neg}$ & Neg / Pos & $2+$ & $\mathrm{Neg}$ & Pos & I & Pos \\
\hline & & Corpus: $1+$ & Corpus: $0+$ & $\mathrm{Neg}$ & Neg / Pos & & & & & \\
\hline \multirow[t]{2}{*}{13} & Pos & Antrum: 1+ & Antrum: 0+ & Pos & Pos / Pos & $1+$ & $\mathrm{Neg}$ & NA & / & NA \\
\hline & & Corpus: NA & Corpus: NA & Pos & Pos / Neg & & & & & \\
\hline \multirow[t]{2}{*}{14} & Pos & Antrum: NA & Antrum: NA & NA & NA / NA & $1+$ & Neg & Pos & I & $\mathrm{Neg}$ \\
\hline & & Corpus: NA & Corpus: NA & NA & NA / NA & & & & & \\
\hline
\end{tabular}

Neg=negative result. Pos=positive result. Inflammation grade: $0+=$ no inflammation; $1+=$ mild inflammation; $2+=$ moderate inflammation; $3+=$ severe inflammation. H. pylori histology: $0=$ no bacteria found; $1+=$ low density of bacteria; $2+=$ moderate density of bacteria; $3+=$ high density of bacteria. H. pylori PCR: G- genus pair of primers; S- species pair of primers. NA= data not available.

\section{Histology}

For histological identification of H. pylori in paraffin sections of sinus tissue, the modified Warthin-Starry silver staining kit (Merck, Germany) was used.

The grading of the histological material, as well as all the molecular biology procedures, were performed after the identification of the samples was masked.

\section{Statistics}

Statistical analysis employed descriptive statistics and the Spearman's rank correlation coefficient (Spearman's rho).

\section{Results}

The study group included 8 patients with asthma, 6 with allergic rhinitis, and 2 with intolerance to nonsteroidal anti-inflammatory drugs (NSAIDs). Other co-morbidities included: hypertension $(n=2)$, hypothyroidism $(n=1)$, goiter $(n=1)$, angioma of the liver $(n=1)$, ankylosing spondylitis $(n=1)$, fibromyalgia while on NSAID's $(n=1)$, gastroesophageal reflux disease (GERD) $(n=2)$ and chronic gastritis $(n=1)$. Two patients were undergoing revision sinus surgery, and three admitted having taken an oral antibiotic in the immediate three months prior to surgery. One subject had history of $H$. pylori gastric infection treatment, while five 
Table 2. Statistically significant correlations between the different variables tested.

\begin{tabular}{|c|c|c|c|}
\hline \multicolumn{2}{|c|}{ Variables } & $\begin{array}{l}\text { Correlation } \\
\text { Coefficient }\end{array}$ & $p=$ value \\
\hline PCR-G results in the nose & PCR-G results in the antrum & 0.655 & 0.040 \\
\hline PCR-S results in the nose & Previous anti- H. pylori treatment & 0.677 & 0.011 \\
\hline Breath test & Cultural identification of H. pylori in the stomach & 0.625 & 0.030 \\
\hline Cultural identification of $H$. pylori in the stomach & Inflammatory status in the antrum & 0.802 & 0.005 \\
\hline Histological identification of H. pylori in the antrum & Inflammatory status in the antrum & 0.769 & 0.009 \\
\hline Histological identification of H. pylori in the antrum & Histological identification of $H$. pylori in the corpus & 0.732 & 0.025 \\
\hline Breath test & PCR-G results in the corpus & 0.655 & 0.040 \\
\hline Histological identification of H. pylori in the corpus & PCR-G results in the corpus & 0.936 & 0.001 \\
\hline Cultural identification of $H$. pylori in the stomach & PCR-G results in the corpus & 0.816 & 0.004 \\
\hline Cultural identification of $H$. pylori in the stomach & PCR-G results in the antrum & 0.828 & 0.002 \\
\hline Histological identification of H. pylori in the corpus & PCR-G results in the antrum & 0.763 & 0.017 \\
\hline PCR-G results in the antrum & PCR-G results in the corpus & 1 & - \\
\hline
\end{tabular}

were taking a PPI until the day before surgery. The patient with ankylosing spondylitis was under treatment with sulfasalazine at the time of the surgical procedure, and the stiffness of his entire spine was so severe that the gastroenterologist was unable to perform the required gastroduodenal endoscopy, even under general anesthesia. Another patient underwent upper gastrointestinal endoscopy outside of the time frame imposed by the strict study criteria (either intra-op or up to two weeks post sinus surgery), so the gastric results were excluded from the analysis. Two patients had histology results for one gastric site only, and in one patient the gastric histological results were altogether absent.

The inflammatory status of the sinus mucosa at the time of surgery for each patient is displayed in Table 1. Severe inflammation was not encountered in any subject; moderate inflammation was found in $50 \%(n=5)$ of the asthmatics and in 16.6 $\%(n=1)$ of the non-asthmatics, whilst mild inflammation was encountered in $50 \%(n=4)$ of the asthmatics and in $83.3 \%(n=5)$ of the non-asthmatics. A total of $60 \%(n=3)$ of patients in the moderate sinus inflammation group had allergic rhinitis, while mild inflammation was observed in $50 \%(n=3)$ of the patients with a diagnosis of allergic rhinitis. All patients with intolerance to NSAIDs in the study group displayed moderate sinus inflammation.

Moderate sinus inflammation was also encountered in $66.7 \%$ $(n=2)$ of the patients who had taken an antimicrobial agent in the three months prior to surgery, whilst mild inflammation was mostly found $(72.7 \%, n=8)$ in patients who had not recently needed antibiotic treatment.

The only patient who had prior treatment for $H$. pylori gastric infection displayed moderate sinus inflammation, while, among the five patients who were on PPI's at the time of surgery, three showed mild sinus inflammation and two moderate sinus inflammation.

Table 1 shows the results of the gastric and nasal inflammatory and H. pylori infection status for all patients.

All patients were found to have some degree of gastric inflammation, fitting the histopathological diagnosis of chronic non-atrophic gastritis (active or non-active), even when the endoscopist failed to identify inflammation and performed iterative biopsies. A total of $60 \%$ of the antrum biopsies showed moderate inflammation, with mild inflammation occurring in $40 \%$, whereas in corpus biopsies $70 \%$ had mild and $30 \%$ moderate inflammation.

Histological identification of $H$. pylori in gastric mucosa biopsies of the antrum was negative in $50 \%$ of the cases $(n=5)$, revealing mild infection in $10 \%(n=1)$, moderate infection in $10 \%(n=1)$, and severe infection in $30 \%$ of the cases $(n=3)$. For the corpus biopsies, the results were negative in $50 \%(n=5)$, with mild infection in $10 \%(n=1)$, moderate in $30 \%(n=3)$, and severe infection in $10 \%(n=1)$.

The results of cultures of gastric mucosa biopsies were positive for $H$. pylori in $66.7 \%$ of the cases $(n=8)$, whilst the PCR-G test was positive in $54.5 \%(n=6)$ of the antrum samples and in $50 \%$ $(n=5)$ of the corpus samples, and the PCR-S test was positive in $72.7 \%(n=8)$ of the antrum samples and in $66.7 \%(n=8)$ of the corpus samples.

A total of $66.6 \%$ of the patients receiving PPI treatment were $H$. pylori negative.

Table 2 shows the correlations between the variables found to 
have statistical significance.

Regarding $H$. pylori presence in the sinonasal mucosa, the species-specific PCR identification was positive in only two cases (15.4\%), although $69.2 \%$ had sinonasal samples with positive genus-specific PCR results. The first case of positive H. pylori DNA in the sinuses had a positive breath urea test and tested positive for $H$. pylori in the stomach by histology, bacterial culture and PCR. The second case, however, had a negative breath test and tested negative for gastric $H$. pylori by histology and bacterial culture, but the species-specific PCR identification test in the stomach was positive (result confirmed at a different laboratory). All attempts to grow $H$. pylori in culture medium from sinonasal samples failed.

\section{Discussion}

The characteristics of our investigation, with patients undergoing simultaneous extensive sinus and stomach tissue sampling, and with various tests concurrently performed, necessarily restricts the number of patients enrolled in the study, and as a result may not be able to show statistical significance. The available data, however, are sufficient to allow for important conclusions.

Since urease production is a hallmark of active gastric infection, it is perhaps no surprise that most, but not all, patients in our study with a positive urease breath test had indeed $H$. pylori gastric infection, confirmed by histopathology, culture, and/or PCR testing. The breath test was positive in one case where $H$. pylori was detected in the sinuses, but was negative in the other. As the majority of patients in our study had positive breath tests and no evidence of $H$. pylori in their sinuses, this apparently renders the test unsuitable for the specific identification of the bacterium in the nose.

Our data actually reveals that, in spite of the relevant number of patients with positive Helicobacter-genus DNA detected by PCR in the sinus mucosa (which certainly merits separate investigation), specifically H. pylori DNA was only found in the sinonasal mucosa of about $15.4 \%$ of chronic rhinosinusitis surgical patients. These results are in agreement with previously published data ${ }^{(3-15)}$. Admittedly, this prevalence could indeed be greater if the bacterium in the nose would follow a similar mosaic pattern of mucosal infection as in the stomach, with patches of diseased mucosa alternating with non-infected normal mucosa, in which case it would require repeated and extensive sampling to allow for a positive $H$. pylori result (five different sites, according to some) ${ }^{(20)}$.

Both cases with H. pylori DNA in the sinuses had H. pylori DNA simultaneously present in their stomachs, but only about 25$28.6 \%$ of the patients with positive gastric species-specific PCR tests had H. pylori DNA in their sinuses. This suggests that if the bacterium is to be encountered in the nose, its DNA has to also be present in the stomach, but that not all H. pylori gastric infections are necessarily associated with $H$. pylori colonization of the sinuses.

Our data show that while there is a positive correlation between cultural and histological identification of $H$. pylori in the stomach and gastric inflammation, we found no such correlation between $\mathrm{H}$. pylori and site inflammation in the nose (Table 2). Therefore, it is perhaps not farfetched to admit that $H$. pylori presence in the nose and sinuses does not contribute to local mucosal inflammation.

The diagnosis of allergic rhinitis, asthma or intolerance to NSAIDs, was not found to statistically relate to either positive or negative sinonasal $H$. pylori results, suggesting that sinonasal $H$. pylori colonization may occur regardless these co-morbidities are present or not.

Critically, H. pylori could only be recovered from the nose in the DNA form, as all the attempts to culture the bacterium from nasosinusal sites failed. This inability to culture H. pylori could be due to the presence of too few microorganisms to be detected, or the simultaneous presence of too many types of other bacteria in the nose that inhibit growth of H. pylori. However, it has been shown that the bacterium can be cultured from adverse environments such as the air sampled during vomiting or from a tracheostomy tube ${ }^{(2)}$. So it is admissible that the reason may not have to do with the method but with the possibility that, in the sinuses, either the organism is represented just by fragments of its DNA and that these are destined to transiently remain there just for a limited time, or the microorganism is, in fact, in a dormant state that precludes culture, and is destined to remain for a long time in the sinuses.

The bacterium is, indeed, known to be able to resist harsh environments by changing to a dormant, inactive state, a non-culturable coccoid form that could still be potentially viable, later on, in the stomach ${ }^{(21,22)}$. It is therefore possible that $H$. pylori may lay dormant for long periods of time, using the nose and the sinuses as reservoirs, waiting for an eventual return to an active form, either to cause gastric re-infection or to participate in the oral-oral route of transmission. The fact that in a previous study no statistical difference was observed between $H$. pylori nasal colonization in patients with sinusitis when compared to the control group ${ }^{(6)}$, lends credibility to the 'nose as a reservoir' thesis, and reinforces our conviction that the bacterium's presence in the nose does not contribute to the local inflammatory status. To account for the bacterium's presence in the nose, the hypothesis of gastric-nasal transmission seems the most logical explanation since a significant number of patients with GERD and laryngopharyngeal reflux (LPR) also have $H$. pylori gastric colonization. It has been shown that the microorganism has a positive tropism for mucins ${ }^{(23)}$ - and mucins cover and protect the sinus and mouth epithelia ${ }^{(24)}$ - and is also able to invade epithelial cells ${ }^{(25)}$. The presence of $H$. pylori in the sinuses could then be regarded as a biomarker of the extent of LPR in the upper 
airway tract. However, at this time, we have no definitive proof of this, and we simply cannot rule out the possibility that the bacterium may use, in alternative or in conjunction, other routes to have its DNA reach the sinuses, for instance, via lymphatic or vascular transmission, from either the stomach or any other extra-digestive site.

Also, the presence of $H$. pylori in the sinuses apparently does not support a local pathogenic role does not entirely rule out the possibility that the bacterium may influence the course of an inflammatory disease of the sinuses. H. pylori gastric infection is known to cause a vast array of systemic effects, including a strong immunologic response and gastrin and cytokine release from the stomach mucosa, all of which may indirectly affect chronic inflammation in any part of the respiratory system ${ }^{(2)}$. Definitive proof of the clinical relevance of these $H$. pylori-induced systemic effects is, however, still lacking.

\section{Conclusion}

Our results suggest that, regardless of how $H$. pylori reaches the sinuses, its presence there does not seem to contribute to the local inflammatory status of the respiratory mucosa. The fact that H. pylori could not be cultured from nose samples and is only present in its DNA form, suggests either a transient presence of parts of its genome in the sinuses, or, instead, what could be seen as a defensive adaptive reaction in preparation for a more or less lengthy stay at an inhospitable location, a change to a viable non-culturable form, from which $H$. pylori could hypothetically regain activity, to either play a role in the oral-oral route of transmission or in an eventual gastric re-infection.

\section{Acknowledgements}

We thank Dr. Sérgio Dinis for assisting with the statistical analysis, Dra. Mónica Oleastro for confirming the PCR results, and Dra. Patrícia Fonseca for reviewing the manuscript.

\section{Authorship contribution}

PBD: Concept and design, data acquisition and analysis, drafting and final approval of the manuscript, accountability for all aspects of the work. TM: Data acquisition and analysis, final approval of the manuscript. MS: Data acquisition and analysis, draft revision, final approval of the manuscript. PLA: Data acquisition and analysis, draft revision, final approval of the manuscript. JV: Data acquisition and analysis, final approval of the manuscript. AMC: Data acquisition and analysis, draft revision, final approval of the manuscript. JV: Concept and design, data acquisition and analysis, drafting, final approval of the manuscript, accountability for all aspects of the work.

\section{Conflict of interest}

The Jorge Vitor's lab has received funding from New England Biolabs Inc., USA, since 1995. All the other authors have no conflicts of interest to disclose.

\section{References}

1. Payão SLM, Rasmussen LT. Helicobacter pylori and its reservoirs: A correlation with the gastric infection. World J Gastrointest Pharmacol Ther 2016; 7: 126-132.

2. Malfertheiner MV, Kandulski A, Schreiber J, Malfertheiner P. Helicobacter Pylori Infection and The Respiratory System: A Systematic Review Of The Literature. Digestion 2011; 84: 212-220.

3. Ozdek A, Cirak MY, Samim E, Bayiz U, Safak MA, Turet S. A possible role of Helicobacter pylori in chronic rhinosinusitis: a preliminary report. Laryngoscope 2003; 113: 679-682.

4. Morinaka S, Ichimiya M, Nakamura $\mathrm{H}$. Detection of Helicobacter pylori in nasal and maxillary sinus specimens from patients with chronic sinusitis. Laryngoscope 2003; 113: 1557-1563.

5. Koc C, Arikan OK, Atasoy P, Aksoy A Prevalence of Helicobacter pylori in patients with nasal polyps: a preliminary report. Laryngoscope 2004; 114: 1941-1944.

6. Dinis PB, Subtil J. Helicobacter pylori and Laryngopharyngeal Reflux in Chronic Rhinosinusitis. Otolaryngol Head Neck Surg 2006; 134: 67-72

7. Cvorovic L, Brajovic D, Strbac M, Milutinovic Z, Cvorovic V. Detection of Helicobacter pylori in nasal polyps: preliminary report. J Otolaryngol Head Neck Surg 2008; 37 :

\section{2-195.}

8. Kim HY, Dhong HJ, Chung SK, Chung KW Chung YJ, Jang KT. Intranasal Helicobacter pylori colonization does not correlate with the severity of chronic rhinosinusitis. Otolaryngol Head Neck Surg 2007; 136 390-395.

9. Ozyurt M, Gungor A, Ergunay K, Cekin E Erkul E, Haznedaroglu T. Real-time PCR detection of Helicobacter pylori and virulence-associated cagA in nasal polyps and laryngeal disorders. Otolaryngol Head Neck Surg 2009; 141: 131-135.

10. Burduk PK, Kaczmarek A, Budzynska A Kazmierczak W, Gospodarek E. Detection of Helicobacter pylori and cagA gene in nasal polyps and benign laryngeal diseases. Arch Med Res 2011; 42: 686-689.

11. Jelavic B, Grgić M, Cupić H, Kordić $M$, Vasilj M, Baudoin T. Prognostic value of Helicobacter pylori sinonasal colonization for efficacy of endoscopic sinus surgery. Eur Arch Otorhinolaryngol 2012; 269: 21972202.

12. Včeva A, Danić $D, V c ̌ e v ~ A$, et al. The sig nificance of Helicobacter pylori in patients with nasal polyposis. Med Glas (Zenica) 2012; 9: 281-286.

13. Nikakhlagh $S$, Samarbafzadeh AR, Jahani M, et al. Determining the Role of Helicobacter pylori in Chronic Sinus Infections Using the
Polymerase Chain Reaction. Jundishapur J Microbiol 2015; 21;8:e20783.

14. Bansal D, Sharma S, Agarwal S, Saha R Gupta N. Detection of Helicobacter pylori in Nasal Polyps. Head Neck Pathol 2016; 10:306-313.

15. Leason SR, Barham HP, Oakley G, et al. Association of gastro-oesophageal reflux and chronic rhinosinusitis: systematic review and meta-analysis. Rhinology 2017; 55:3-16.

16. Lund VJ, Kennedy DW. Quantification for staging sinusitis. Ann Otol Rhinol Laryngol 1995; 104 (Suppl 167):17-21.

17. Snidvongs K, Lam M, Sacks $R$, et al. Structured histopathology profiling of chronic rhinosinusitis in routine practice. Int Forum Allergy Rhinol 2012; 2:376-385.

18. Moyaert $H$, Pasmans F, Ducatelle $R$, Haesebrouck F, Baele M. Evaluation of $16 S$ rRNA gene-based PCR assays for genuslevel identification of Helicobacter species. J Clin Microbiol 2008; 46: 1867-1869.

19. Menard A, Santos A, Mégraud F, Oleastro M. PCR-Restriction Fragment Length Polymorphism Can Also Detect Point Mutation A2142C in the 23S rRNA Gene, Associated with Helicobacter pylori Resistance to Clarithromycin. Antimicrob Agents Chemother 2002; 46: 1156-1157.

20. Garza-González E, Perez-Perez GI, 
Maldonado-Garza HJ, Bosques-Padilla FJ. A review of Helicobacter pylori diagnosis, treatment, and methods to detect eradication. World J Gastroenterol 2014; 20: 14381449

21. Azevedo NF, Almeida C, Cerqueira L, Dias S, Keevil CW, Vieira MJ. Coccoid Form of Helicobacter pylori as a Morphological Manifestation of Cell Adaptation to the Environment. Appl Environ Microbiol 2007; 73: 3423-3427.

22. Reshetnyak VI, Reshetnyak TM. Significance of dormant forms of Helicobacter pylori in ulcerogenesis. World J Gastroenterol 2017 23: 4867-4878.

23. Celli JP, Turner BS, Afdhal NH, et al.
Helicobacter pylori moves through mucus by reducing mucin viscoelasticity. Proc Nat Acad Sci U S A 2009; 106: 14321-14326.

24. Ali ME-S, Pearson J P. More Than One Disease Process in Chronic Sinusitis, Based on Mucin Fragmentation Patterns and Amino Acid Analysis. Int J Otolaryngol 2015; 13: 1-8.

25. Phillipson M, Johansson MEV, Henriksnas J, et al.. The gastric mucus layers: constituents and regulation of accumulation. Am J Physiol Gastrointest Liver Physiol 2008; 295: G806-G812.
Paulo Borges Dinis, MD

Otolaryngology Department

CHLN- Hospital Santa Maria

Av Prof Egas Moniz

1649-035 Lisboa

Portugal

Tel: +351919091226

E-mail: borges.dinis2@gmail.com 